\title{
CIENCIA\&SALUD
}

\section{Microbiota intestinal y su influencia en el comportamiento.}

\section{Intestinal microbiota and behavior influence.}

Karla Chaves Morales' María Catalina Camacho Alvarado²

\author{
1 Médico general. Caja costarricense de seguro social, Alajuela Costa Rica \\ 2 Médico general. Caja costarricense de seguro social, San José Costa Rica \\ Contacto: kchavesm137@gmail.com mariacata.camacho@gmail.com
}

\section{Cómo citar:}

Chaves Morales, K., \& Camacho Alvarado, M. C. Microbiota intestinal y su influencia en el comportamiento. Revista Ciencia Y Salud, 6(1), Pág. 49-56. https://doi. org/10.34192/cienciaysalud. v6i1.374

Recibido: 29/Sep/2021

Aceptado: 24/Ene/2022

Publicado: 14/Feb/2022

\section{RESUMEN}

El microbioma humano se refiere al conjunto de microorganismos que residen en el cuerpo, mientras que la microbiota se refiere a la comunidad ecológica de un nicho o sistema específico. Su transmisión puede ocurrir de forma vertical, desde los padres a la descendencia y de manera horizontal por las interacciones sociales y la cohabitación; además puede variar por condiciones propias del individuo. Se considera que la microbiota intestinal tiene influencia en varios procesos cerebrales, a través del ahora conocido eje intestino-cerebro (EIC). Este se conforma por el tracto gastrointestinal (TGI), el sistema nervioso entérico (SNE) y el cerebro. Al establecer una simbiosis con la microbiota, el huésped puede depender de la misma para funciones como mielinización, neurogénesis, activación microglial y modulación del entorno. Sin embargo, de forma colateral la composición y la diversidad de la microbiota pueden afectar el comportamiento e influir en procesos como el estrés, la ansiedad y la cognición; y otros desórdenes neurológicos específicos.

Palabras Clave: Microbioma, Microbiota, Intestino, Cerebro, Comportamiento.

\section{ABSTRACT}

The human microbiome refers to the set of microorganisms that reside in the body, while the microbiota refers to the ecological community of a specific niche or system. Its transmission can occur vertically, from parents to offspring, and horizontally through social interactions and cohabitation; it may also vary due to the individual's own conditions. The gut microbiota is considered to influence various brain processes, through the now known gut-brain axis. This is made up of the gastrointestinal tract, the enteric nervous system and the brain. By establishing a symbiosis with the microbiota, the host can depend on it for functions such as myelination, neurogenesis, microglial activation and modulation of the environment. However, collaterally, the composition and diversity of the microbiota can affect behavior and influence processes such as stress, anxiety and cognition and other specific neurological disorders.

\section{Keywords: Microbiome, Microbiote, Gut, Brain, Behavior.}




\section{INTRODUCCIÓN}

El término microbioma fue introducido en el 2001 por el biólogo molecular estadounidense Joshua Ledenberg, quien declaró que los microorganismos simbióticos y los humanos forman una unidad metabólica (1). Las raíces griegas de la palabra microbioma significan micro: pequeño, y biome: vida; y se define como el conjunto de microorganismos y sus productos génicos: ácido desoxirribonucléico (ADN), ácido ribonucleico (ARN) y metabolitos. Se considera que está compuesto por 90 billones de bacterias, arqueas u organismos unicelulares, microorganismos eucariotas y virus (2).

Gracias al estudio del microbioma humano se amplía la teoría tradicional en la que uno o varios microorganismos patógenos son responsables de un proceso infeccioso. Ahora se contempla que el ambiente humano es un ecosistema complejo, donde la pérdida del equilibrio en sus distintos nichos biológicos genera enfermedades y susceptibilidades inmunológicas (2).

La microbiota se refiere a la comunidad de microorganismos que residen en un hábitat específico, con poblaciones de especies estables y otras variables (3). Los proyectos Microbioma Humano (HMP por sus siglas en inglés) y Metagenómica del Tracto Intestinal Humano (MetaHIT) establecieron los primeros catálogos de genes microbianos de la microbiota humana adulta. Cada hábitat tiene una variación relativamente baja cuando se compara entre individuos sanos de una misma distribución geográfica, raza, origen étnico y fase de la vida (2).

Clásicamente, la labor y el parto se catalogan como la primera y mayor exposición al complejo microbiota. Este es un mecanismo primordial de transferencia intergeneracional de microbioma en los mamíferos, conocido como transmisión vertical, extendiéndose directamente de los padres a la descendencia (4). Sin embargo, existe evidencia que sugiere que la primera exposición a microorganismos ocurre en la vida intrauterina. La exposición a factores estresantes durante la gestación altera el microbioma materno. Esto promueve respuestas inflamatorias y traslocación bacteriana a la placenta, conlleva cambios neuroinflamatorios fetales y provoca diferencias en el neurodesarrollo que persisten en el adulto (5).

Las bacterias neonatales preparan el desarrollo de sistemas inmune, metabólico, hormonal y nervioso. En condiciones naturales, la leche materna tiene una implicación notable en el desarrollo sensorial y motor al contener microbiota comensal. Procesos como la dentición y maduración del sistema gastrointestinal permiten recibir alimentos sólidos aumentando la diversidad $(4,6)$.

La transmisión horizontal de microbioma comprende las interacciones sociales y cohabitación. También está determinada por factores propios en el individuo como: la dieta que a largo plazo corresponde al determinante primario de la microbiota humana intestinal; la genética del huésped, ejercicio, infecciones, estrés y ciclos de sueño. Una de las formas más dramáticas de influir el microbioma corresponde al uso de antibióticos, aún en ciclos cortos, dado que los genes de resistencia pueden persistir en la comunidad microbiana después del término de la terapia $(6,7)$.

\section{MATERIALES Y MÉTODOS}

La literatura utilizada en la redacción de este documento fue obtenida mediante búsqueda en PubMed y Cochrane Database, con palabras en idiomas español e inglés, como: microbioma, microbiota, intestinal, intestino, comportamiento, social, influencia, alimentos, dieta, enfermedad neurológica y cerebro. Se brindó prioridad a información actualizada en los últimos cuatro años.

\section{DESARROLLO}

La microbiota humana establece una relación simbiótica con el hospedero, la colonización ocurre en piel, vías respiratorias, sistema reproductivo, oral y tracto gastrointestinal. Aporta funciones de protección ante patógenos por modulación de la respuesta inmune. En la piel produce péptidos antimicrobianos y 


\section{CIENCIA\&SALUD}

ácidos orgánicos que reducen el pH, funcionando como barrera, variando según la temperatura, humedad, distribución glandular y profundidad de la piel. En el tracto respiratorio, previamente considerado estéril, varía en vía respiratoria alta y baja; y evita el avance de patógenos a lo largo del mismo. En el caso de la mujer, el tracto reproductivo está compuesto principalmente por lactobacilos que se asocian con un estado saludable, acidifican el entorno y actúan de barrera ante la colonización de otros microorganismos $(2,8)$.

El potencial oxidación-reducción, gradientes químicos y nutricionales, inmunidad del huésped y la capa mucosa son algunos de los factores asociados a la variabilidad espacial en la composición de la microbiota en el TGI. Se cree que el esófago alberga algunas bacterias y levaduras, probablemente provenientes de la orofaringe por la deglución o gástricas por reflujo gastroesofágico (9). A nivel estomacal, el pH ácido del estómago previene el crecimiento bacteriano, aunque desde los años ochenta se reconoce la colonización por Helicobacter pilory, donde estudios de secuenciación de ARN confirman la presencia de distintos filotipos en biopsias gástricas (2).

El intestino delgado y el intestino grueso constituyen la región con mayor densidad microbiana, poseen papeles metabólicos en producción de vitaminas, nutrientes esenciales y de protección, al actuar como barrera contra las infecciones. Asimismo, posee un efecto modulador inmune endocrino y actúa en la secreción de neurotransmisores que comunican el intestino y el cerebro $(5,8)$. Existen diversas técnicas por las cuales se ha estudiado la composición intestinal. Entre las más relevantes se encuentran el cultivo microbiano, microscopía electrónica, secuenciación de segunda generación, metagenómica (caracterización de todos los genes de un nicho), metaproteómica (caracterización de proteínas) y metabolómica (caracterización de metabolitos). Por medio de ellas, se conoce la presencia de bacterias, arqueas, hongos, virus y bacteriófagos (9).

El concepto de biogeografía explica cómo los compartimentos intestinales cuentan con una comunidad miocrobiana distinta, presentándose una mayor diversidad de proximal hacia distal; es decir, se cuenta con menor número a nivel de duodeno y yeyuno que en íleon distal y colon (2). Es probable que esto corresponda de manera directamente proporcional a la disponibilidad de nutrientes. A su vez, la composición varía de manera importante entre individuos, lo cual dificulta la posibilidad de establecer una microbiota sana de referencia, aunque se habla de cuatro familias dominantes: Bacteroidetes, Firmicutes, Proteobacteria, y Actinobacteria (9).

La bidireccionalidad de la microbiota se ha estudiado ampliamente al trasplantar complejos fecales en ratones libres de gérmenes. De esta forma, al realizar el trasplante de un donador humano sano a un ratón, se estimula la producción de serotonina y se disminuye el tiempo de tránsito intestinal. Por otro lado, al utilizar moduladores del tránsito intestinal como polietilenglicol o loperamida, se observan cambios significativos en la microbiota intestinal de estos roedores (9).

Más allá de la influencia loco regional, la microbiota a su vez puede verse alterada según la experiencia. Emociones y situaciones estresantes pueden causar un cambio en la biodiversidad bacteriana (vía descendente), resultando en un crecimiento preferencial de ciertas comunidades y asimismo, la microbiota puede influenciar procesos del sistema nervioso central (SNC) (vía ascendente). Estos procesos hacen referencia al "eje intestino-cerebro (EIC)" (10,11).

Según investigaciones la señalización del EIC ocurre mediante tres vías principales: el décimo par craneal, el sistema inmune y por metabolitos microbianos. Mediante un modelo murino en 2011, se describe que el principal circuito de modulación entre el TGI y el SNE corresponde al nervio Vago o décimo par craneal, el cual posee divisiones eferentes y aferentes (12). En 2008 se indica que la simbiosis bacteriana es crucial en la maduración del sistema inmune y que este sistema provee otra ruta de comunicación entre los gérmenes y el cerebro (13). En información más reciente, se ha reconocido que las bacterias intestinales contribuyen al metabolismo del hospedero por la producción de metabolitos, detallados más adelante, que influyen en las funciones fisiológicas (14). 


\section{CIENCIA\&SALUD}

La división aferente del nervio Vago conecta el TGI con el núcleo del tracto solitario (NTS) y niveles superiores de control emocional del cerebro mamífero. Aunque se sabe que la porción sensitiva del nervio Vago guarda una relación directa con el SNE, se desconocen los mecanismos específicos de señalización (10). Se cree que el nervio Vago tiene la capacidad de censar señales microbianas mas no realiza una interacción directa con la microbiota. Estudios sugieren que existe una modulación mediada por células enteroendocrinas y enterocromafines del epitelio gastrointestinal. Las bacterias intestinales producen metabolitos como butirato, propionato, acetato y valerato, los cuales se asocian a las funciones de motilidad, secreción e inflamación intestinal (9).

Tejidos en el cerebro y el intestino son derivados del ectodermo en el desarrollo fetal y comparten vías de señalización, incluyendo los neurotransmisores y neuropéptidos. La serotonina es uno de los reguladores más importantes en el eje intestino-cerebro-microbioma; regula funciones conductuales y fisiológicas incluyendo la ansiedad, entorno, vigilancia, agresión, humor, impulsividad, sueño y apetitito (15).

Los ácidos grasos de cadena corta, productos del metabolismo bacteriano, estimulan la enzima triptófanohidroxilasa, desencadenando producción de serotonina (5-HT) en las células enterocromafines. Aferencias vagales envían señales al NTS y al núcleo Dorsal del Rafé, donde se encuentra la mayoría de neuronas serotoninérgicas a nivel cerebral. A su vez, se producen señales que viajan hacia centros superiores de control emocional y de regulación del estado de ánimo (10). Otros neurotransmisores implicados en las vías de señalización corresponden al cortisol, norepinefrina y dopamina (15).

El eje hipotálamo-pituitario-adrenal representa otra ruta de influencia del eje intestino cerebro. Regula la respuesta del cuerpo al estrés y su disregulación está asociada con desórdenes de salud mental. Al estudiarlo por primera vez se mostró que los ratones libres de gérmenes tienen una respuesta exagerada en el mismo, lo cual disminuye al suplementarlos con bacterias como Bifidobacterium infantis, revertiendo síntomas depresivos. En otros estudios fueron tratados de forma crónica con Lactobacillus rhamnosus moderando también la ansiedad (16).

El sistema inmune intestinal se encarga de la tolerancia homeostática de organismos comensales y de la protección del cuerpo contra patógenos de manera simultánea. La respuesta inmune a microbios en el SNE está mediada por receptores tipo toll (RTT) y peptidoglicanos donde parte de su función es prevenir una respuesta inmune inapropiada. Algunos productos microbianos como los lipopolisacáridos (LPS) pueden llegar al SNC por medio de la circulación. En modelos animales con roedores, se han encontrado RTT a nivel de la microglia en enfermedades como Alzheimer, Parkinson, dolor y depresión, sugestivos de expresión génica producto de cambios en la microbiota (17).

Pequeñas moléculas producidas por el microbioma pueden causar cambios neurofisiológicos por medio de cuatro vías: sanguínea, humoral, inmunológica y neuronal (18). Se ha estudiado la participación de la microbiota en procesos como mielinización, neurogénesis, activación microglial y modulación del entorno, además de alteración de la neurofisiología del cerebro en regiones clave para el comportamientos social y ansioso, incluyendo amígdala e hipocampo. Al comprometer este último puede alterar el aprendizaje y la memoria $(19,20)$.

Esta red bidireccional de terminaciones entéricas y vagales activadas por metabolitos y sustancias neuroactivas producidas en el lumen intestinal, depende de la traslocación de estas señales desde la mucosa intestinal al flujo sanguíneo y de la barrera hematoencefálica al cerebro $(21,22)$.

La composición del microbioma intestinal difiere entre individuos y es más parecida al comparar la microbiota de un mismo sujeto a lo largo de la vida que entre distintas personas, de un mismo contexto étnico-socio cultural, como se mencionó anteriormente. Dicha composición y su diversidad están asociadas a los cambios de personalidad. Personas con mayor sociabilidad poseen mayor diversidad microbiana, comprobando que el ambiente social puede promover la diversidad, los géneros Akkermansia, Lactococos y Oscillospira 


\section{CIENCIA\&SALUD}

son más abundantes en estos individuos. Los géneros Desulfovibrio y Sutterella fueron más abundantes en individuos menos sociales. A su vez, las personas con menor diversidad se asocian a niveles superiores de estrés y ansiedad (23).

Aquellos que comen alimentos con probióticos y prebióticos naturales tienen niveles significativamente menores de ansiedad y estrés, son menos propensos a sufrir dichas enfermedades en comparación con individuos que los consumen de forma suplementaria (23). En un reporte con voluntarios sanos que recibieron suplementación prebiótica con un producto comercial que contiene galactooligosacaridos por tres semanas, reveló que los participantes mostraron un nivel de vigilancia disminuido a información negativa o positiva con respecto al grupo placebo (24). Los probióticos pueden modular la serotonina en la corteza frontal y los metabolitos dopaminérgicos corticales, disminuyendo los síntomas depresivos; y así pueden mejorar el humor en humanos y el desempeño en test cognitivos (16).

El estrés puede provocar cambios en los hábitos de alimentación, incrementando la sobrealimentación y obesidad, exacerbando síntomas depresivos. La grasa interfiere en la síntesis de serotonina, mientras que las proteínas tienen un efecto opuesto. Se ha hipotetizado que dietas altas en grasa causan desórdenes de humor y dietas similares a las mediterráneas generan un efecto contrario. Estas dietas ricas en vegetales, frutas y nueces, bajo consumo de carnes y consumo moderado de vino tinto, poseen relación con la duración del sueño, asociando a patrones de 6-7 horas por noche con mayor calidad del descanso y reducción del insomnio (15). Se ha evidenciado que las frutas y los vegetales incrementan las sensaciones de satisfacción y felicidad. Las nueces mejoran el humor y reducen la tensión o ansiedad. Los antioxidantes incluidos el folato, vitamina E y omega 3 poseen características neuroprotectoras (25).

El EIC también influye en cambios del comportamiento de desórdenes neurológicos específicos, los más sobresalientes corresponden a: trastornos de espectro autista, enfermedad de Parkinson y enfermedad de Alzheimer (16).

En el desarrollo de trastornos del espectro autista, la genética es un factor clave en la patogénesis, pero se estima que más del $50 \%$ de la neurobiología conduce a factores no hereditarios. Se ha demostrado que niños con autismo han recibido menor consumo de suplementos de fibra y menor lactancia materna. Una manifestación usualmente no reconocida de estos desórdenes es la marcada comorbilidad de síntomas gastrointestinales (16). El trasplante de microbiota intestinal de donadores humanos con autismo a ratones libres de gérmenes reveló que la colonización fue suficiente para inducir un comportamiento autista en los animales (26).

La formación de agregados de la proteína alfa sinucleína corresponde a uno de los pilares en la patología de la enfermedad de Parkinson. Dicha proteína se ha identificado en la mucosa y submucosa de fibras nerviosas y ganglios de pacientes con síndromes parkinsonianos. Alguna evidencia pre clínica sugiere que puede ser transportada al cerebro vía nervio vago. Cuando los ratones fueron colonizados por microbiota de pacientes con Parkinson desarrollaron déficits motores y neuroinflamación (16).

Los cambios en la microbiota de los pacientes con Alzheimer se asocian a mayores concentraciones pro inflamatorias y una reducción de los anti inflamatorios, lo que puede iniciar o exacerbar la neurodegeneración en los pacientes con la enfermedad, sin embargo, esto se ha descrito en pequeños estudios (16). 
Es conveniente que el paciente conozca su condición con el objetivo de tomar decisiones oportunas de alimentación y prevenir condiciones físicas o mentales desfavorables (25). Sin embargo, la genética nutricional es un campo novedoso no implementado aún como parte de la terapéutica de trastornos neurológicos.

\section{CONCLUSIONES}

Pocas enfermedades crónicas humanas pueden explicarse de manera exclusiva por factores genéticos, se conoce la participación de múltiples factores ambientales en el desarrollo y progresión de la enfermedad. El conocimiento de la microbiota intestinal y su interacción en procesos neuroinmunológicos, abre una gran área de estudio y de terapéutica en el futuro. Sin embargo, a pesar de tecnologías que permiten catalogar de manera detallada el conjunto de microorganismos con los cuales las personas conviven, aún no se comprende completamente el mecanismo por el cual pueden interferir en los procesos biológicos humanos.

Se requieren estudios adicionales para determinar con una mayor precisión las consecuencias funcionales de las vías de señalización conocidas y sus implicaciones en el neurodesarrollo, comportamiento y papel en enfermedades agudas y crónicas.

Durante los últimos años el consumo de alimentos ha sufrido cambios debido a la variedad y accesibilidad, provocando efectos positivos y negativos en la salud mental. Desafortunadamente la mayoría de publicidad se enfoca en promover el consumo de alimentos altos en grasa, azúcares y calorías. Aumentar el consumo de alimentos no saludables genera experiencias de estrés, emociones negativas o agresivas. Seleccionar alimentos saludables y variados promueve un estado mental y conductual beneficioso. Por ahora, se puede asegurar que la alimentación es uno de los pilares de la medicina preventiva.

\section{REFERENCIAS BIBLIOGRÁFICAS}

1. Sebastián Domingo JJ, Sánchez Sánchez C. From the intestinal flora to the microbiome. Rev Esp Enfermedades Dig [Internet]. 2017 [citado 20 de diciembre de 2021];110. Disponible en: https:// online.reed.es/fichaArticulo.aspx?iarf=684769749239-413272195161

2. Bennett JE, Dolin R, Blaser MJ. Enfermedades infecciosas: principios y práctica [Internet]. 2021 [citado 20 de mayo de 2021]. Disponible en: https:// auth.elsevier.com/ShibAuth/institutionLogin?entityID=https\%3 A\% 2 F 2 Fwww. rediris.es\%2Fsir\%2Fubidp\&appReturnURL=https\%3 A\%2F\%2Fwww.clinicalkey. com\%2Fstudent\%2Fcontent\%2Ftoc\%2F3-s2.O-C2019004558X

3. Berg G, Rybakova D, Fischer D, Cernava T, Vergès M-CC, Charles T, et al. Microbiome definition re-visited: old concepts and new challenges. Microbiome. diciembre de 2020;8(1):103.

4. Dominguez-Bello MG, Godoy-Vitorino F, Knight R, Blaser MJ. Role of the microbiome in human development. Gut. junio de 2019;68(6):1108-14.

5. Aagaard K, Luna RA, Versalovic J. The Human Microbiome of Local Body Sites and Their Unique Biology. En: Mandell, Douglas, and Bennett's Principles and Practice of Infectious Diseases [Internet]. Elsevier; 2015 [citado 20 de mayo de 2021]. p. 11-8. Disponible en: https://linkinghub.elsevier.com/ retrieve/pii/B9781455748013000023

6. Knight R, Callewaert C, Marotz C, Hyde ER, Debelius JW, McDonald D, et al. The Microbiome and Human Biology. Annu Rev Genomics Hum Genet. 31 de agosto de 2017;18(1):65-86.

7. Bailey MT, Cryan JF. The microbiome as a key regulator of brain, behavior and immunity: Commentary on the 2017 named series. Brain Behav Immun. noviembre de 2017;66:18-22. 


\section{CIENCIA\&SALUD}

8. Requena T, Velasco M. Microbioma humano en la salud y la enfermedad. Rev Clínica Esp. abril de 2021;221(4):233-40.

9. Feldman M, Friedman LS, Brandt LJ, editores. Sleisenger and fordtran's gastrointestinal and liver disease: pathophysiology, diagnosis, management. 11.a ed. Philadelphia: Elsevier; 2020.

10. Cussotto S, Sandhu KV, Dinan TG, Cryan JF. The Neuroendocrinology of the Microbiota-GutBrain Axis: A Behavioural Perspective. Front Neuroendocrinol. octubre de 2018;51:80-101.

11. Johnson KV-A, Foster KR. Why does the microbiome affect behaviour? Nat Rev Microbiol. octubre de 2018;16(10):647-55.

12. Bercik P, Park AJ, Sinclair D, Khoshdel A, Lu J, Huang X, et al. The anxiolytic effect of Bifidobacterium longum NCC3001 involves vagal pathways for gut-brain communication. Neurogastroenterol Motil. diciembre de 2011;23(12):1132-9.

13. Edelman SM, Kasper DL. Symbiotic commensal bacteria direct maturation of the host immune system: Curr Opin Gastroenterol. noviembre de 2008;24(6):720-4.

14. De Vadder F, Kovatcheva-Datchary P, Goncalves D, Vinera J, Zitoun C, Duchampt A, et al. Microbiota-Generated Metabolites Promote Metabolic Benefits via Gut-Brain Neural Circuits. Cell. enero de 2014;156(1-2):84-96.

15. Bremner J, Moazzami K, Wittbrodt M, Nye J, Lima B, Gillespie C, et al. Diet, Stress and Mental Health. Nutrients. 13 de agosto de 2020;12(8):2428.

16. Cryan JF, O'Riordan KJ, Sandhu K, Peterson V, Dinan TG. The gut microbiome in neurological disorders. Lancet Neurol. febrero de 2020;19(2):179-94.

17. Margolis KG, Cryan JF, Mayer EA. The Microbiota-Gut-Brain Axis: From Motility to Mood. Gastroenterology. abril de 2021;160(5):1486-501.

18. Benakis C, Martin-Gallausiaux C, Trezzi J-P, Melton P, Liesz A, Wilmes P. The microbiome-gutbrain axis in acute and chronic brain diseases. Curr Opin Neurobiol. abril de 2020;61:1-9.

19. Cenit MC, Sanz Y, Codoñer-Franch P. Influence of gut microbiota on neuropsychiatric disorders. World J Gastroenterol. 2017;23(30):5486.

20. Vuong HE, Yano JM, Fung TC, Hsiao EY. The Microbiome and Host Behavior. Annu Rev Neurosci. 25 de julio de 2017;40(1):21-49. 


\section{CIENCIA\&SALUD}

21. Forssberg H. Microbiome programming of brain development: implications for neurodevelopmental disorders. Dev Med Child Neurol. julio de 2019;61(7):744-9.

22. Lavazza A, Sironi VA. Are we Ready for a "Microbiome-Guided Behaviour" Approach? Camb Q Healthc Ethics. octubre de 2019;28(04):708-24.

23. Johnson KV-A. Gut microbiome composition and diversity are related to human personality traits. Hum Microbiome J. marzo de 2020;15:100069.

24. Mohajeri MH, La Fata G, Steinert RE, Weber P. Relationship between the gut microbiome and brain function. Nutr Rev. 1 de julio de 2018;76(7):481-96.

25. AlAmmar WA, Albeesh FH, Khattab RY. Food and Mood: the Corresponsive Effect. Curr Nutr Rep. septiembre de 2020;9(3):296-308.

26. Sharon G, Cruz NJ, Kang D-W, Gandal MJ, Wang B, Kim Y-M, et al. Human Gut Microbiota from Autism Spectrum Disorder Promote Behavioral Symptoms in Mice. Cell. mayo de 2019;177(6):16001618.e17. 